\title{
Nefronia lobar aguda derecha secundaria a apendicitis aguda no perforada: reporte de caso
}

\author{
María Angélica Wilches-Cuadros* \\ Stefania Roca-López** \\ Luis Miguel Sosa***
}

*Médico general. Facultad Ciencias de la Salud. Universidad Autónoma de Bucaramanga. Bucaramanga. Colombia.

**Médico general. Residente II año de Pediatría. Departamento de Pediatría. Escuela de Medicina. Facultad de Salud. Universidad Industrial de Santander. Bucaramanga. Colombia.

***Médico Pediatra Especialista en Infectología Pediátrica. Profesor de Cátedra. Departamento de Pediatría. Escuela de Medicina. Facultad de Salud. Universidad Industrial de Santander. Bucaramanga. Colombia.

Correspondencia: Dr. María Angélica Wilches. Dirección: Cra 11 \# 119-39, Edificio La Capilla Apt 203, Bogotá DC. Colombia. Teléfono: 3016895634.

Correo electrónico: mwilches@unab.edu.co

\section{Resumen}

La apendicitis aguda en pediatría ocurre generalmente en escolares y adolescentes, siendo su principal complicación el absceso intraabdominal. Por su parte, la nefronía lobar aguda es una infección localizada del parénquima renal, de la cual no existen casos reportados de ésta como secundaria a apendicitis no perforada. Se presenta el caso de una preadolescente, quien seis días después de una apendicectomía consulta por dolor abdominal, vómito y fiebre. Ingresa con signos de respuesta inflamatoria sistémica, por lo que se inicia terapia antibiótica, y luego de tres días sin mejoría clínica, se realiza un ecografía abdominal que evidencia una lesión renal derecha sugestiva de nefronía lobar aguda y un absceso hepático del segmento VI y VII. Se inicia terapia antibiótica de amplio espectro con adecuada respuesta clínica. La nefronía lobar aguda debe tenerse en cuenta entre las complicaciones de la apendicitis, dado su comportamiento agresivo, rápida progresión a sepsis y frecuente asociación a cicatrices renales. MÉD.UIS.2019;32(1):33-7

Palabras clave: Dolor abdominal. Nefritis. Apendicectomía. Complicaciones Posoperatorias. Infecciones Intraabdominales.

\section{Acute right lobar nephronia secondary to acute non-perforated appendicitis: a case report}

\section{Abstract}

Acute appendicitis in pediatrics presented in scholar and adolescent ages have as its main complication an intraabdominal abscess. Moreover, acute lobar nephronia is a localized infection in the renal parenchyma. There are no cases reported about nephronia as a consequence of acute non-perforated appendicitis. This case describes a preadolescent presenting abdominal pain, vomit and fever, six days after acute appendicitis. She was admitted with signs of systemic inflammatory response syndrome, so antibiotic therapy was started. After three days there was no clinical improvement, whereby an abdominal ultrasound was performed, which reported injury in the right kidney suggestive of acute lobar nephronia and an hepatic abscess of VI and VII segments. Broad spectrum of antibiotic therapy was started with adequate clinic response. Acute lobar nephronia should be considered when studying acute appendicitis complications, given it's aggressive behavior, accelerated progression to sepsis and frequent association with renal scarring. MÉD.UIS.2019;32(1):33-7

Keywords: Abdominal Pain. Nephritis. Appendectomy. Postoperative Complications. Intraabdominal Infections. 
¿Cómo citar este artículo?: Wilches-Cuadros MA, Roca-López S, Sosa LM. Nefronía lobar aguda derecha secundaria a apendicitis aguda no perforada: reporte de caso. MÉD.UIS. 2018;32(1):33-7. doi: 10.18273/revmed.v32n1-2019005

\section{Introducción}

La apendicitis aguda es la primera causa de dolor abdominal quirúrgico en población pediátrica en países de mediano a alto ingreso; presentándose principalmente en la segunda década de la vida, con una media entre los 10 y 11 años'. Es también la primera causa de sepsis abdominal en escolares y adolescentes de países de alto ingreso ${ }^{2}$. La perforación ocurre en $10-20 \%$ de los pacientes adolescentes y hasta en $80-$ $100 \%$ de los menores de cuatro años ${ }^{1,3}$. La tasa de complicaciones de la apendicitis es en promedio 10-15\%, siendo la más frecuente el absceso intraabdominal ${ }^{4}$, cuyo riesgo post-operatorio de aparición es del $20 \%$ para apendicitis perforada y del $0,8 \%$ en apendicitis no perforada'. Otras complicaciones frecuentes son obstrucción intestinal, íleo paralítico, infección de herida quirúrgica y dolor. Sin embargo, menos del $1 \%$ requiere una nueva intervención, y la mortalidad es $\operatorname{rara}(0,1 \%)^{4}$.

La nefronía lobar aguda es una infección localizada no licuefactiva del parénquima renal, considerada un intermedio entre la pielonefritis aguda y el absceso renal $^{5}$. Al igual que las infecciones de vías urinarias, es principalmente causada por bacterias gram negativas 5 . Se desconoce su epidemiología local; sin embargo, una serie de casos realizada en Taiwán reportó una prevalencia de $19,2 \%$ en niños con primer episodio de infección urinaria ${ }^{6}$.

Actualmente, no existen casos descritos de la nefronía lobar aguda como complicación de la apendicitis, por lo que es poco tenida en cuenta en el estudio de las complicaciones de la misma, a pesar de ser de gran importancia dada su rápida progresión a sepsis y asociación con secuelas renales como las cicatrices. En este artículo se expone un caso de nefronía lobar posterior a un episodio de apendicitis aguda no perforada.

\section{Caso clínico}

Se presenta el caso de una paciente femenina de diez años quien consultó a urgencias por dolor abdominal de tres días de evolución sin signos de irritación peritoneal, localizado en hemi-abdomen superior, el cual, 24 horas previas a la consulta, migra a fosa ilíaca derecha; asociado a un episodio emético. Ante la sospecha de apendicitis aguda es llevada a cirugía, donde la descripción intraoperatoria refiere apendicitis flegmonosa con drenaje de líquido seropurulento a cavidad abdominal, con reporte de patología de apendicitis edematosa, por lo que recibió terapia antibiótica hospitalaria con metronidazol y amikacina durante cinco días, y al egreso con cefalexina oral por siete días.

36 horas después del egreso consulta nuevamente por fiebre de $39^{\circ} \mathrm{C}$, dolor punzante en hemi-abdomen derecho y siete episodios eméticos biliosos. Se halló al examen físico de ingreso presión arterial limítrofe (89/62 mmHg), taquicárdica (137 latidos/minuto), taquipnéica (34 respiraciones/minuto), febril $\left(38,3^{\circ} \mathrm{C}\right)$, con saturación limítrofe ( $90 \%$ ambiente) y glucometría normal $(90 \mathrm{mg} / \mathrm{dl})$. Se encontraba alerta, en posición antálgica, sin aspecto tóxico, con abdomen doloroso a la palpación generalizada superficial y profunda, sin irritación peritoneal. Debido a los hallazgos, se decidió hospitalizar por sospecha de absceso intraabdominal. Recibió reanimación endovenosa con cristaloides, se le tomaron de exámenes y se reinició manejo antibiótico con amikacina y metronidazol.

Los laboratorios reportaron leucocitosis (20.800/ $\mathrm{ul})$, neutrofilia $(17.100 / \mathrm{mm} 3)(82 \%)$, hematocrito

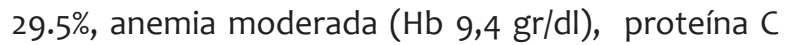
reactiva elevada ( $361 \mathrm{mg} / \mathrm{l})$; mientras que el recuento de plaquetas (475.000/ul), la función renal y el examen de orina se encontraron normales. De igual forma, el urocultivo y dos hemocultivos tomados fueron negativos. Por otra parte, la ecografía de abdomen mostró aumento del volumen renal bilateral, principalmente derecho (riñón derecho $108 \times 51 \times 51 \mathrm{~mm}$, corteza $7,8 \mathrm{~mm}$ y riñón izquierdo de $98 \times 55 \times 55 \mathrm{~mm}$, corteza de $12 \mathrm{~mm}$ ), sin masas o quistes y sin cambios en los demás órganos.

Luego de 72 horas de tratamiento, la paciente no presentaba dolor abdominal, pero permanecía febril, con vómito ocasional y signos de respuesta inflamatoria sistémica. Nuevos exámenes 
documentaron aumento de la leucocitosis (25.000/ ul), neutrofilia $(20.570 / \mathrm{mm} 3)$, empeoramiento de la anemia (Hb $8.8 \mathrm{gr} / \mathrm{dl}$ ), trombocitosis (666.000/ ul) y PCR persistentemente elevada (378 mg/l). Su función renal y radiografía de tórax eran normales. Los dos hemocultivos permanecían negativos. En una segunda ecografía abdominal se observó una lesión hipo-ecoica heterogénea dependiente del polo superior del riñón derecho de 58×57 mm, y alteración del espacio pararenal anterior, sugestivo de nefronía lobar derecha. Además, presentaba cambios en la ecogenicidad de lóbulo hepático derecho, en los segmentos VI y VII, de 55×43 mm, compatible con absceso hepático.
Por lo anterior, se inició vancomicina y meropenem. Luego de siete días, la paciente se encontraba afebril, sin dolor y sin signos de respuesta inflamatoria sistémica. Sus laboratorios eran normales, excepto por la PCR de 64 mg/l. A los catorce días, una gammagrafía con ácido dimercaptosuccínico demostró cambios cicatriciales en polo superior y un proceso inflamatorio focal en polo superior y tercio medio de riñón derecho. La función renal era normal. La paciente completó 21 días de terapia antibiótica, y en la ecografía control se observó disminución de aproximadamente 50\% de la lesión inicial (Ver Figura 1), lo cual, sumado a que la paciente se encontraba asintomática, permitió que fuera dado el egreso médico.
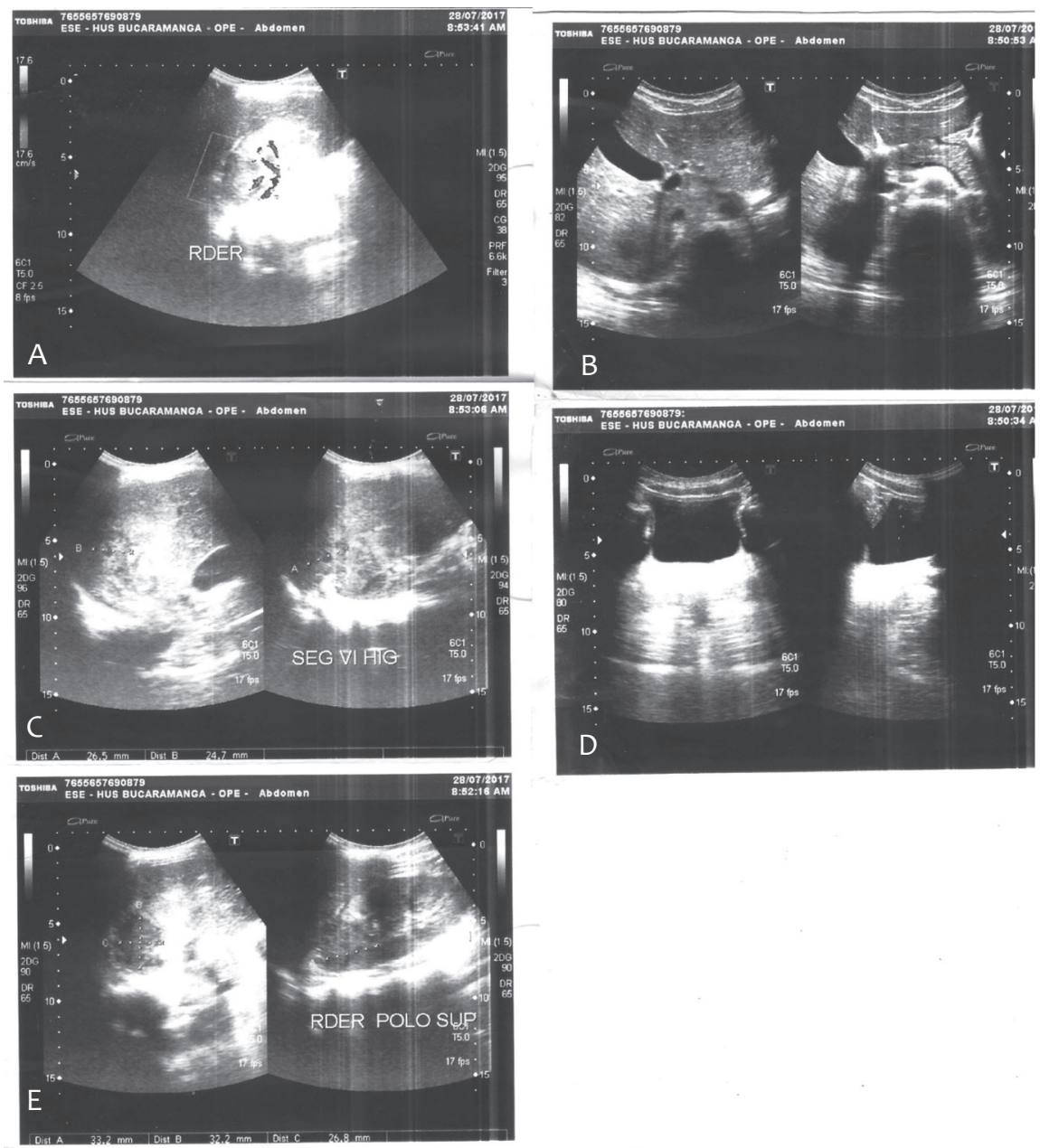

Figura 1. Ecografía de control después de 21 días de tratamiento.

A - E. Área hipo-ecoica con el polo superior del riñón derecho de 33×26 mm en los bordes lateral y transverso. No hay flujo Doppler en esta área. No hay otras lesiones en el resto del parénquima renal. B-C. Área hipo-ecoica poco definida en el segmento VI hepático con un tamaño de $26 \times 24 \mathrm{~mm}$. No existen colecciones o lesiones estructurales asociadas. No hay dilatación de la vía biliar intra o extra hepática. La vesícula biliar se encuentra normalmente distendida, sin cálculos o cambios inflamatorios en sus paredes. D. La vejiga se encuentra adecuadamente dilatada con paredes regulares sin defectos endoluminales.

Fuente: Autores. 


\section{Discusión}

En la apendicitis aguda el tiempo de evolución de los síntomas previos a la consulta se asocia a mayor riesgo de complicaciones, pues este aumenta casi cinco veces [OR 4,9] si la duración es mayor a 48 horas y casi quince veces [OR 14,6] si es mayor a 72 horas $^{7,8}$. Sin embargo, no aumenta con el tiempo entre la atención médica y la apendicectomía9,10. Se ha descrito un mayor riesgo, inconsistente en algunos estudios, con un recuento de leucocitos mayor a 15.000/ul [OR 16,38 $]^{9,11}$. En el caso de la paciente, la evolución del dolor fue de aproximadamente 72 horas y presentó un recuento de leucocitos de $20.800 / u l$, lo cual, acorde a lo descrito en la literatura, aumenta su riesgo de complicaciones.

Se debe administrar una dosis peri-operatoria de antibiótico de amplio espectro en todos los casos de apendicitis con el fin de reducir la incidencia de infección de herida quirúrgica y absceso intraabdominal $^{12}$. Si en cirugía se observa perforación, se debe administrar terapia combinada como ceftriaxona y metronidazol o monoterapia con piperacilina/ tazobactam ${ }^{1,12}$. La duración del tratamiento farmacológico es guiada clínicamente por la resolución de la fiebre, desaparición del dolor, función intestinal y el recuento de leucocitos'. Se recomienda tratar sietediez días en promedio ${ }^{12}$, aunque no hay diferencia en complicaciones post-quirúrgicas con ciclos terapéuticos cortos (tres-cinco días) ${ }^{13}$. En el caso, aunque la apendicitis era no perforada, el equipo quirúrgico decidió extender la terapia antibiótica debido a la presentación del cuadro con signos de respuesta inflamatoria sistémica y el hallazgo de líquido seropurulento en cavidad.

A pesar de la conducta anteriormente referida, la paciente presentó como complicación nefronía lobar y absceso hepático. Se considera que esta complicación pudo haber sucedido por la extensión vía linfo-hematógena de la apendicitis aguda, a través de la vena mesentérica superior o sistema linfático ileo-cecal, dejando siembras a nivel renal y en los segmentos VI y VII hepáticos; o por vía directa, teniendo en cuenta que intra-operatoriamente se encontró drenaje de líquido seropurulento en cavidad abdominal, pudiendo por extensión peritoneal causar siembras a nivel renal y hepático. De acuerdo con la literatura, la nefronía suele ocurrir tradicionalmente como extensión por vía ascendente de una infección de vías urinarias bajas en un paciente con factores de riesgo de ascenso (como el reflujo vesico-ureteral).

Por otra parte, la nefronía lobar tiene curso clínico agresivo, comparada con la pielonefritis, dado que la fiebre persiste por más tiempo luego de iniciado el tratamiento, presenta mayor leucocitosis, elevación de la PCR y trombocitopenia ${ }^{14}$. El paciente suele tener aspecto tóxico, malestar generalizado, escalofríos, fiebre y vómito. En escolares progresa rápidamente hacia la sepsis, mientras que en niños mayores prevalecen síntomas localizados como dolor en flancos ${ }^{5,14}$. En el caso descrito la nefronía se presentó con mayor compromiso del estado general del paciente que la apendicitis aguda, aunque inicialmente se consideró como primera posibilidad un absceso intraabdominal. Es posible, por el hallazgo de líquido seropurulento en cavidad y tiempo de evolución del dolor, que la apendicitis estuviera en una fase más avanzada y por ende mayor probabilidad de diseminación. De igual manera, cabe la posibilidad de que la paciente presentara una infección del tracto urinario concomitante; sin embargo, no fueron tomados urocultivos debido a que ya había recibido más de siete días de terapia antibiótica, y aun así, fue enviada con estudios ambulatorios para descartar reflujo vesico-ureteral, sin embargo el seguimiento de la paciente no fue posible por red de acceso limitada.

El diagnóstico de la nefronía es mediante imágenes, observando en la ecografía una lesión hipoperfundida, de márgenes irregulares y alteración de la relación cortico-medular. Además, en fases tempranas, se puede observar edema del polo renal ${ }^{5}$. Por otra parte, algunos estudios sugieren que nefromegalia severa o nefromegalia moderada asociada a fiebre mayor a 72 horas, tiene $90 \%$ de sensibilidad y $86,4 \%$ de especificidad para el diagnóstico. Además, la presencia de masa aumenta la sensibilidad hasta 95\%5,14. A pesar de que la tomografía de abdomen es el estándar de oro5, en este caso, los hallazgos ecográficos y la presentación clínica fueron considerablemente sugestivos de nefronía, por lo que se consideró no realizar la tomografía para evitar el riesgo de irradiación de la paciente.

Por otra parte, esta entidad presenta alta incidencia de cicatrices renales ( $88 \%$ vs $34,8 \%$ comparada con 
pielonefritis), las cuales están relacionadas con predisposición y riesgo futuro de enfermedad renal crónica ${ }^{14}$. Además, se asocia a 15 veces más riesgo de cicatrices [OR 15,1; $p<0,001$ ] independiente de la edad y tratamiento ${ }^{15}$. En este caso, la gammagrafía realizada encontró cambios cicatriciales, lo cual pone en riesgo futuro a la paciente de presentar enfermedad renal crónica. Se desconoce el reporte de la gammagrafía control que fue solicitado para evaluar la presencia de lesiones residuales.

Finalmente, en otros casos reportados es inusual la presentación luego de los 10 años y su coexistencia con otros procesos infecciosos. Solo en un caso, en un adolescente, se documentó neumonía y epidermólisis bullosa asociada a la aparición de nefronía lobar aguda $^{16}$. Sin embargo, no existe un caso en que haya un proceso infeccioso intraabdominal causal como la apendicitis aguda, tal como ocurrió en el presente reporte.

\section{Conclusión}

En el presente caso se expone una enfermedad subdiagnosticada, con una epidemiología no clara, como posible complicación de una apendicitis aguda no perforada, lo que pudo ser un factor de confusión para orientar una sospecha diagnóstica adecuada. No hay casos previamente descritos de esta asociación. Este caso exalta la importancia de tener en cuenta la nefronía lobar aguda como posible complicación de la apendicitis aguda, dado su comportamiento agresivo, rápida progresión a sepsis y frecuente asociación a cicatrices renales. Estudios adicionales son necesarios para describir el comportamiento epidemiológico de esta entidad y su relación como posible complicación de infecciones intra-abdominales.

\section{Referencias bibliográficas}

1. Rentea RM, St. Peter SD. Pediatric appendicitis. Surg Clin North Am. 2017;97(1):93-112.

2. Hadley GP. Intra-abdominal sepsis-epidemiology, aetiology and management. Semin Pediatr Surg. 2014;23(6):357-62.

3. Bundy DG, Byerley JS, Liles EA, Perrin EM, Katznelson J, Rice HE, et al. Does This Child Have Appendicitis? JAMA. 2007;298(4):438-51.

4. Rentea RM, St. Peter SD. Contemporary management of appendicitis in children. Adv Pediatr. 2017:64(1):225-51.

5. Bibalo C, Apicella A, Guastalla V, Marzuillo P, Zennaro F, Tringali C, et al. Acute lobar nephritis in children: not so easy to recognize and manage. World J Clin Pediatr. 2016;5(1):136-42.

6. Yang CC, Shao PL, Lu CY, Tsau YK, Tsai IJ, Lee PI, et al. Comparison of acute lobar nephronia and uncomplicated urinary tract infection in children. J Microbiol Immunol Infect. 2010;43(3):207-14.

7. Singh M, Kadian YS, Rattan KN, Jangra B. Complicated appendicitis: analysis of risk factors in children. Afr J Paediatr Surg. 2014;11(2):109-13.

8. Bonadio W, Brazg J, Telt N, Pe M, Doss F, Dancy L, et al. Impact of in-hospital timing to appendectomy on perforation rates in children with appendicitis. J Emerg Med. 2015;49(5):597-604.

9. Pham XBD, Sullins VF, Kim DY, Range B, Kaji AH, de Virgilio $\mathrm{CM}$, et al. Factors predictive of complicated appendicitis in children. J Surg Res. 2016;206(1):62-6.

10. Serres SK, Cameron DB, Glass CC, Graham DA, Zurakowski D, Karki M, et al. Time to appendectomy and risk of complicated appendicitis and adverse outcomes in children. JAMA Pediatr. 2017;171(8):740-6.

11. Poudel R, Bhandari TR. Risk factors for complications in acute appendicitis among paediatric population. JNMA J Nepal Med Assoc. 2017;56(205):145-8.

12. Lee SL, Islam S, Cassidy LD, Abdullah F, Arca MJ. Antibiotics and appendicitis in the pediatric population: an american pediatric surgical association outcomes and clinical trials committee systematic review. J Pediatr Surg. 2010;45(11):21815

13. Van Rossem CC, Schreinemacher MH, Treskes K, Van Hogezand RM, Van Geloven AA. Duration of antibiotic treatment after appendicectomy for acute complicated appendicitis. Br J Surg. 2014;101(6):715-9.

14. Sheu JN. Acute lobar nephronia in children. Pediatr Neonatol. 2015;56(3):141-2.

15. Cheng CH, Tsau Y, Chang CJ, Chang YC, Kuo CY, Tsai IJ, et al Acute lobar nephronia is associated with a high incidence of renal scarring in childhood urinary tract infections. Pediatr Infect Dis J. 2010;29(7):624-8.

16. Granados Molina A, Espino Hernández M, Gancedo Baranda A, Albillos Merino JC, Álvarez-Cortinas JF, Molina Amores C. Nefronía focal aguda bacteriana: diagnóstico, tratamiento y evolución. An Pediatr (Barc). 2007;66(1):84-6. 